\title{
Wavelet Analysis for Estimation of Mean-Curve of Impulse Waveforms Superimposed by Noise, Oscillations and Overshoot
}

\author{
L. Satish and B. I. Gururaj
}

\begin{abstract}
This paper describes a novel approach to estimate the mean-curve of impulse voltage waveforms that are recorded during impulse tests. These waveforms in practice are superposed by noise, oscillations, and overshoot. The approach is based on multiresolution signal decomposition (a kind of wavelet transform) and has many advantages over existing methods, since it does not assume any model for estimating the mean-curve, is interactive in nature, suitable for full and chopped impulses, does not introduce distortions due to its application, is easy to implement and does not call for changes to existing standards. Results presented show its applicability.
\end{abstract}

Index Terms-Digital processing, IEC 60060-1, IEC 61083-2, impulse testing, mean-curve, multiresolution signal decomposition, wavelet transform.

\section{INTRODUCTION}

$\mathbf{I}$ MPULSE testing of high voltage power apparatus is essential to assess the overall insulation integrity and design adequacies to withstand overvoltage stresses often encountered during service. International [1] and IEEE [2] specify the relevant waveshapes to be used during the impulse test, and these impulses have historically been characterized by parameters such as, peak magnitude, front-time, tail-time, and time-to-chop (in case of chopped wave). Broad guidelines have also been included in the standards for evaluating these quantities from oscillographic traces. While these are fairly easy to use when the impulses are nearly ideal, problems arise when they have superimposed oscillations or overshoot. Standards stipulate that the parameters are to be determined from a mean-curve that needs to be determined graphically by the operator (as judged by the eye). This graphical procedure was used all over the world without problems when cathode-ray oscilloscopes were used for recording the impulse waveforms.

However, in recent years, with the introduction and widespread use of digital waveform recorders and powerful computers in high voltage measurements and testing, a new dimension has emerged. Although it is true that this paradigm shift from analog to digital domain offers great many advantages, it must be acknowledged that it has also created newer challenges. One example of direct relevance is the contrast between manual determination of the impulse waveform

Manuscript received October 16, 1999.

L. Satish was with the High Voltage Institute, H. U. T., Finland. He is now with the Department of High Voltage Engineering, Indian Institute of Science, Bangalore, 560012 India.

B. I. Gururaj is an independent Consultant in Bangalore, India.

Publisher Item Identifier S 0885-8977(01)02231-2. parameters from oscillographic traces to the need to specify mathematical procedures (algorithms) that can satisfactorily translate the currently prevalent guidelines existing in the standards.

The task of developing algorithmic procedures has attracted a lot of attention from researchers and, perhaps, one of the first efforts in this direction happens to be the use of model-based curve fitting for full impulses by McComb and Lagnese [3]. They postulated a mean-curve composed of a sum of two or more exponential functions and even some with quadratic terms in the exponent. Proceeding on similar lines, Li et al. [4] propose double-exponential models (sum of two terms only) along with four fitting algorithms for analyzing full impulses.

Perez and Martinez [5], [6] propose the extended Kalman filtering approach (using the smooth impulse model as proposed in [3]) for constructing the mean-curve. Use of different windows (digital filtering) to remove noise and oscillations has been discussed by Dias, et al. [7]. Oliveria and Miranda [8] demonstrate how the mean-curve could be obtained by filtering the data using Savitzky-Golay filters. A wavelet based approach has also been recently reported in [9], which will be further discussed at the end.

Recently, CIGRE WG 33-03 undertook a comprehensive study to compare existing impulse parameter evaluation procedures [10] wherein a mention has been made that definitions and procedures contained in IEC and IEEE standards are inadequate for the evaluation of impulse parameters while using digital data and further go on to state that, this is the primary reason for proliferation of different interpretations in the estimation of impulse parameters thereby leading to large-scale deviations during inter-comparison studies.

Ten laboratories within Europe compared their individual procedures for evaluating the impulse parameters and the results are published in [11] wherein it has been remarked that impulses with superposed noise, oscillations and overshoot can lead to errors larger than the specified limits. A critical analysis of the above mentioned literature indicates that they all suffer from one or more of the following deficiencies:

1) Impulses occurring in practice deviate considerably from this model, for example, those recorded during impulse tests on transformers, reactors, etc. This matter has not been examined until now in the literature. So, in a strict sense, enforcing a model on the obtained data is not always the correct approach. This has been recognized by McComb and Lagnese [3] who report that it was not possible to get best fit with only one model for all types of 
data. Thus, model-based postulation of mean-curve will require selection of one from many types of functions to get best fit for any given data and hence cannot be a general approach.

2) Performance of most techniques is critically dependent on selection of the interval of data samples (from the front and tail regions) used for fitting the models.

3) In most methods, presence of noise and oscillations greatly affect the performance (convergence failure) and significantly influence the final results. Therefore, most methods recommend noise removal by filtering in the first pass (moving average, digital filtering). This has adverse effects, since it is accompanied by distortion of the impulse (results in flattening on the wave-front portion).

4) Existing methods do not address the task of identification of the superposed oscillations and deviations in a direct manner.

5) In most methods, only mathematical minimization of deviations is discussed, with no mention of physical basis for the fitting being performed.

Bearing these requirements and deficiencies of the existing methods in mind, the authors propose an entirely new method for the purpose of determining the mean-curve, based on the principles of Multiresolution Signal Decomposition (MSD). The approach is based on wavelet transforms, but when the basis functions are chosen to be orthonormal, it guarantees perfect reconstruction of original signal from the individual decomposed components. The great advantage of this method is that there exists an elegant algorithm. Additionally, it is a time-frequency transform and so one has simultaneous information of both the time and frequency distributions of the input signal. This indeed is helpful in the present context, because it can lead to easy identification of both frequency and time of occurrence of the various constituents of the input signal, which in turn can help in the identification of the frequency of superposed oscillations and duration of overshoot, as per the standards. Removal of high frequency noise from the data is simple, as will be shown later, and most importantly introduces no significant distortions in the waveform within the regions of our interest. Removal of superposed oscillations and overshoot can be done interactively by the user, and once the mean-curve is obtained, simple interpolation can be used to compute the impulse parameters. This approach does not require postulation of a model for the mean-curve and so it is applicable to any signal. To the best knowledge of the authors, such a route to determination of mean-curve has not been investigated earlier (The authors became aware of [9] after this work was informally presented to CIGRE WG 33-03 at its meeting in Joensuu, Finland, in August 1998). The paper is organized as follows. After a brief outline of the proposed approach, the results and discussion on analytical signals and those measured during actual impulse tests are presented. Conclusions emerging from this study are given at the end.

\section{OUTLINE OF THE APPROACH}

Wavelet transform (WT) is a powerful signal processing tool for computing time-frequency representation of signals. In multiresolution signal decomposition (MSD) technique, instead of continuous dilation and translation as in WT, a discrete dyadic-orthonormal basis function is used. This is primarily responsible for its improved properties when compared to the normal wavelet transform; like absence of information redundancy, guarantees perfect reconstruction, and a fast algorithm for its computation. The property of perfect reconstruction is very essential in the present work, since we are interested in first identifying the superimposed frequency components and then proceeding to remove them. So, it is essential that the decomposition reconstruction process is error free. The application of WT and MSD for fault detection during impulse testing of transformers, has already been reported in [12], [13].

The MSD procedure involves obtaining low-pass approximations and band-pass detailed-signals from an input signal. An approximation or smoothed-signal is a low resolution representation of the original signal while a detailed signal is the difference between two successive low resolution representations. These approximations and detailed signals are obtained through filter bank structures, using analysis and synthesis filter pairs. Depending on the depth of decomposition required (i.e., number of stages) the process can be continued. A signal sampled at a frequency of $f s$, can be decomposed into $N$ stages using the MSD procedure. The decomposed components are related to the original signal by the following equation:

$$
\text { Input }=(\text { smoothed }- \text { signal })_{N}+\sum_{i=1}^{N}(\text { detailed }- \text { signal })_{i} .
$$

For example, if $N=3$, MSD results in the following signal components:

- $\mathrm{d} 1$, represents frequencies fs $/ 2$ to $\mathrm{fs} / 4$

- $\mathrm{d} 2$, represents frequencies $\mathrm{fs} / 4$ to $\mathrm{fs} / 8$

- $\mathrm{d} 3$, represents frequencies fs/8 to fs/16

- $\mathrm{s} 3$, represents frequencies fs/16 to DC

Hence, using MSD one has information regarding what range of frequency components exist, its starting positions, duration and also its absolute magnitudes thereby making the task of identification of superimposed oscillations and overshoot that much easier.

The implementation of MSD involves choice of a mother wavelet which plays a significant role on final outcome. The choice depends on the nature and kind of signal to be extracted or detected, and in the present work it primarily involves the detection and localization of low amplitude, short-duration, decaying and oscillating signals. One of the popular wavelets shown to be suitable for such applications is the Daubechies' wavelet. The number of coefficients generating the mother wavelet can be increased to get more localized representations in both time and frequency domains. In this study, the symmetrical version of Daubechies' wavelet (symlets) has been employed. The underlying principle of the proposed method can now be summarized as follows.

- The input signal is decomposed by MSD into two sets of signals, namely the detailed-components and smoothedcomponent. 

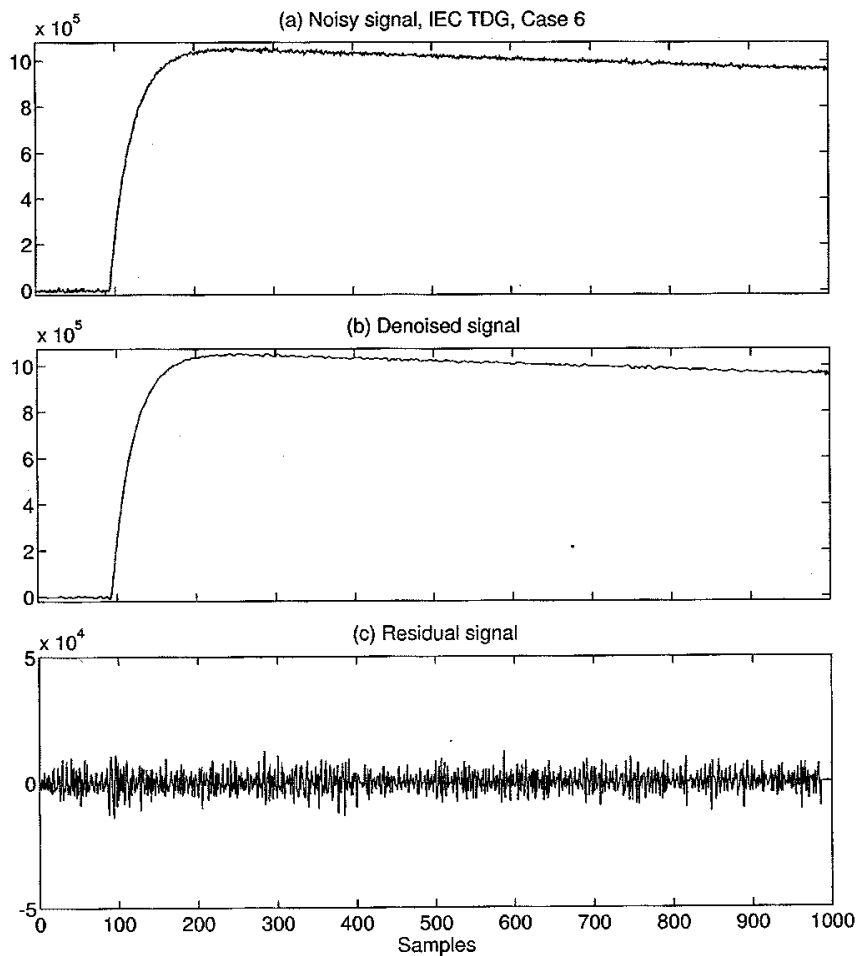

Fig. 1. Removal of high frequency noise.

- A GUI interface can be created to aid the operator to chose those signal components he considers must be suppressed. Mean-curve can be updated and displayed in real-tune, and thus guide the operator in making the right choice.

- The above step is repeated until a satisfactory mean-curve (got by directly summing the remaining components) is obtained.

- Impulse parameters of the mean-curve are computed by simple interpolation.

\section{RESULTS AND DISCUSSION}

The proposed method was first tested with mathematically generated data and found to give correct results. To show that removal of high frequency noise (arising due to quantization, digitizer internal noise, etc.) can easily be done, consider the signal shown in Fig. 1(a) and drawn from IEC 61 083-2 (Case 6, IEC-TDG). This is an analytical data with noise $( \pm 0.4 \%)$. Use of moving average methods to remove this noise can introduce distortions into the signal, especially on wave-front, which has to be avoided at all costs. However, using the present approach, it is not only simple to eliminate such noise, but also it does not introduce any distortions, and importantly needs no additional processing effort. The de-noised and residual signal are shown in parts (b) and (c) of Fig. 1.

Next, consider the signal drawn from IEC-TDG (Case 11) and shown in Fig. 2(a). This is a measured signal and has oscillations on the front and peak regions. Choosing eight levels (or stages) of decomposition, the input is decomposed and Fig. 3 shows the eight detailed-signal components (d1-d8) and one smoothedsignal component (s8).The frequency band represented by each detailed-signal decrease from $\mathrm{d} 1$ to $\mathrm{d} 8$. Therefore, it is natural
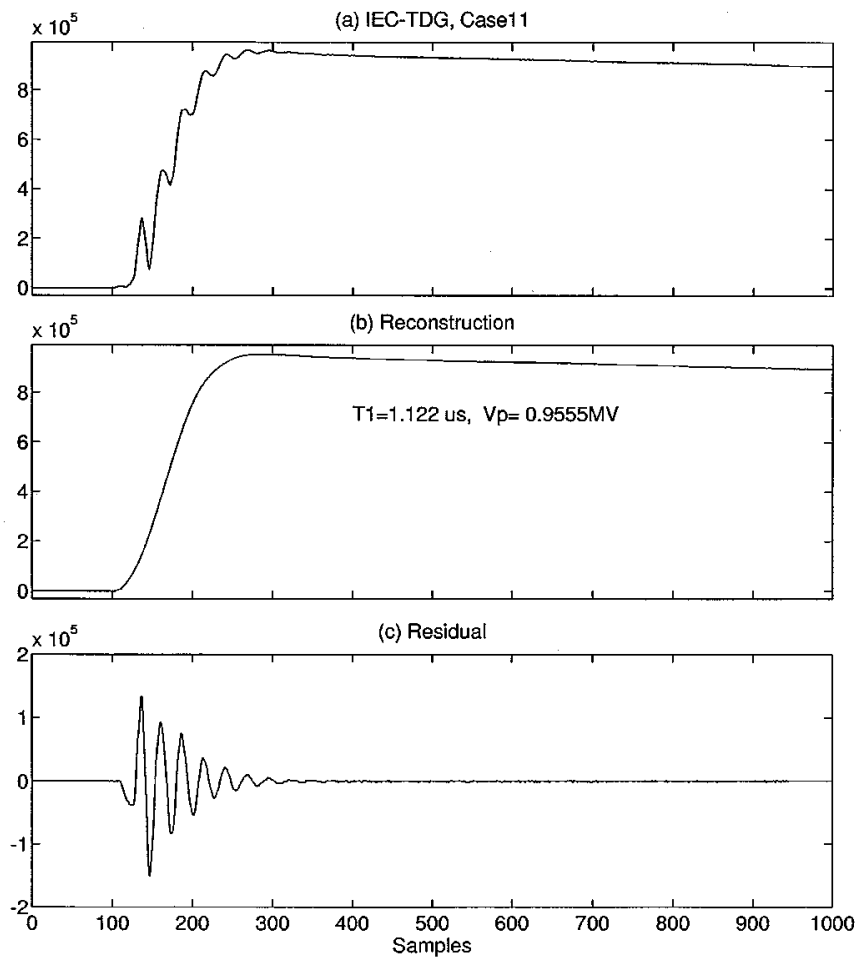

Fig. 2. Removal of front oscillations.

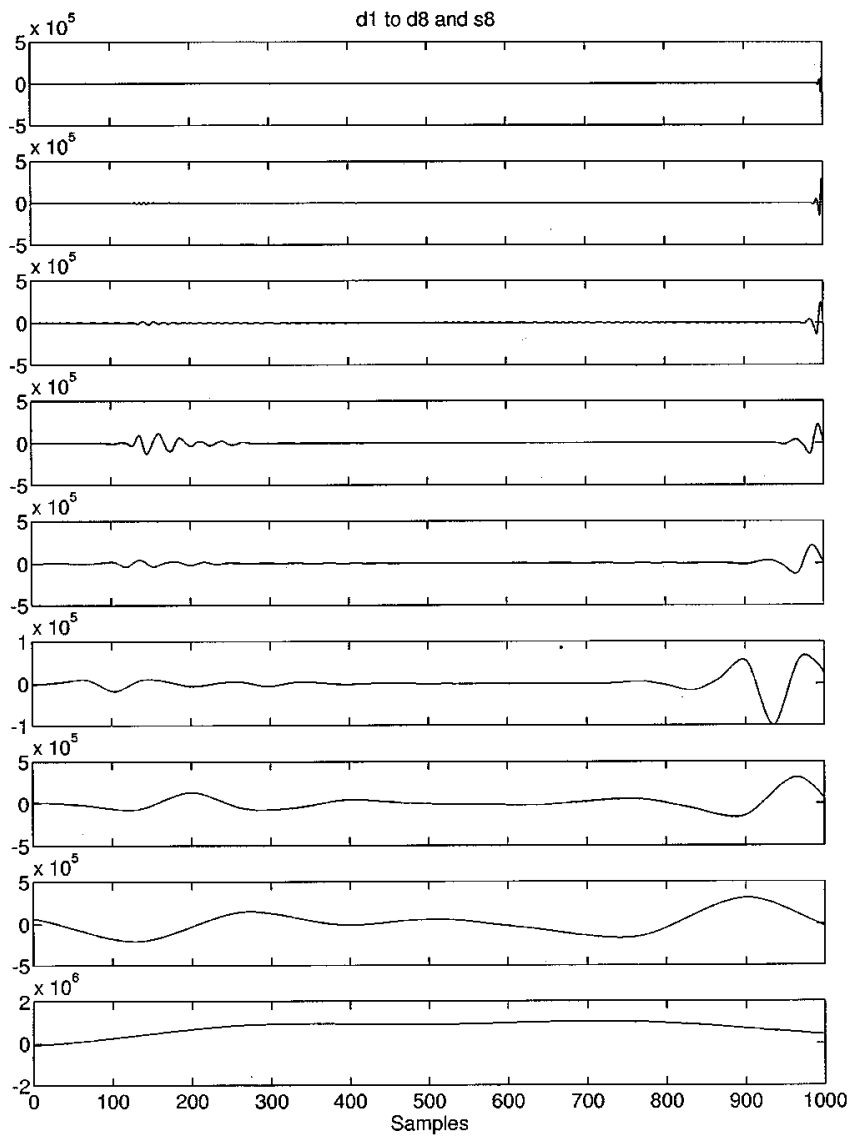

Fig. 3. Eight level MSD of the input signal in Fig. 2(a).

to expect any high frequency noise in the input signal to show up in first one or two levels of the detailed-signals. This can 

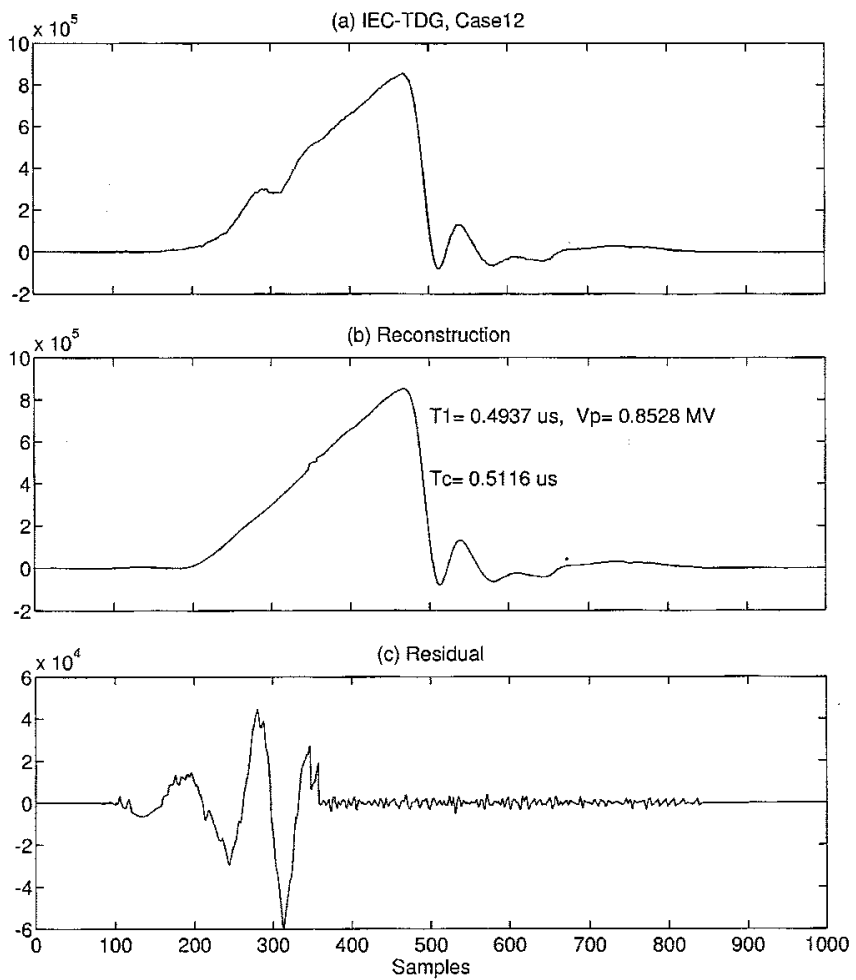

Fig. 4. Removal of front oscillations from a chopped impulse.

easily be identified and removed. It is seen from Fig. 3 that signal components $\mathrm{d} 3, \mathrm{~d} 4$ and $\mathrm{d} 5$, within the time interval of 110-500 samples correspond to the superposed oscillation in the input signal. Direct removal of these portions from d3, d4, $\mathrm{d} 5$, and summing the remaining ones, yields the mean-curve estimate that is shown in Fig. 2(b). The residual signal is shown in Fig. 2(c). The mean-curve appears to be very good. Using simple interpolation, the impulse parameters were computed and are shown in Fig. 2. They are well within the tolerances prescribed.

Fig. 4(a) shows a practically measured chopped impulse with front oscillations (IEC-TDG, Case 12). The meancurve obtained is depicted in Fig. 4(b) along with the residual curve in Fig. 4(c). It is important to note that the processing has successfully removed the front oscillations, and has not introduced any other distortion to the rest of the signal, especially peak value, or the rising and falling edges which is important to determine the chopping-time and also the magnitude of the chop voltage. Such localized processing is an advantage of this method not afforded by conventional digital filtering methods. The impulse parameters computed are shown in Fig. 4, and are well within the specified tolerances.

Next consider Fig. 5(a) which shows a practically measured impulse with long-duration overshoot (Case 13, IECTDG) and noise on the wave-front. The mean-curve obtained is depicted in Fig. 5(b) and the residual curve in Fig. 5(c). The overshoot and the accompanying oscillations have significantly been suppressed. Removal of low frequency phenomenon is comparatively difficult when compared to removal of high frequency phenomenon. The impulse parameters computed are shown in Fig. 5. They are slightly away from the prescribed limits.
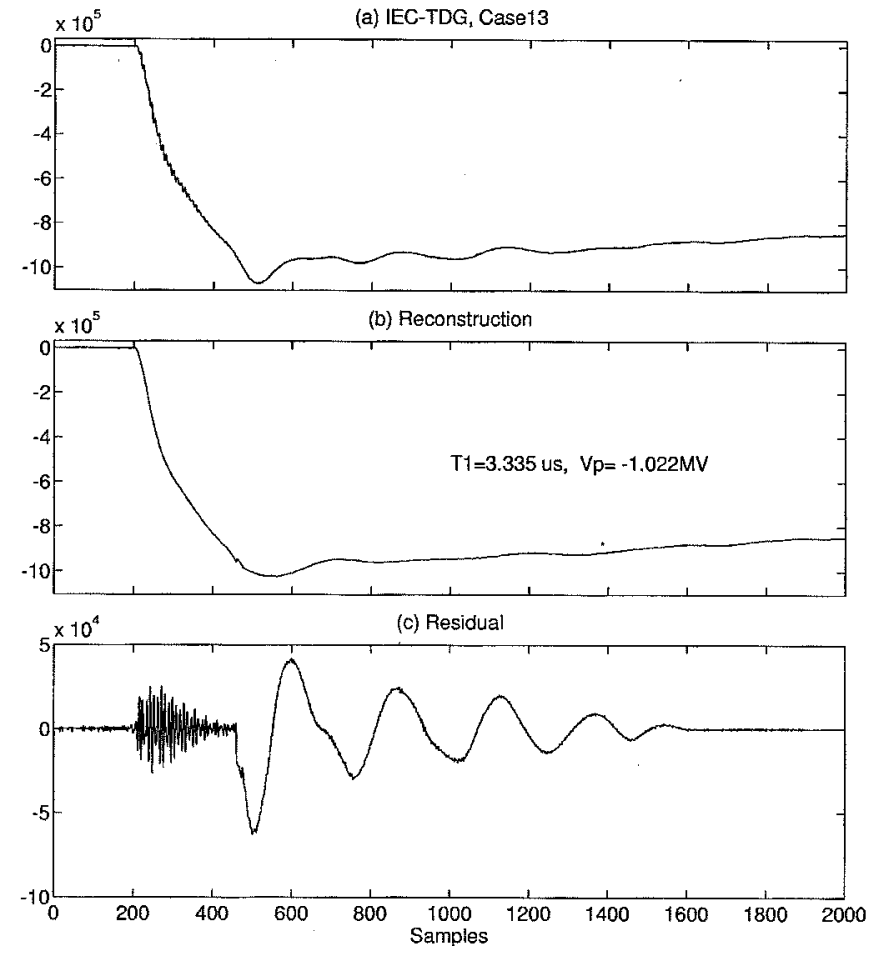

Fig. 5. Isolation of long-duration overshoot.
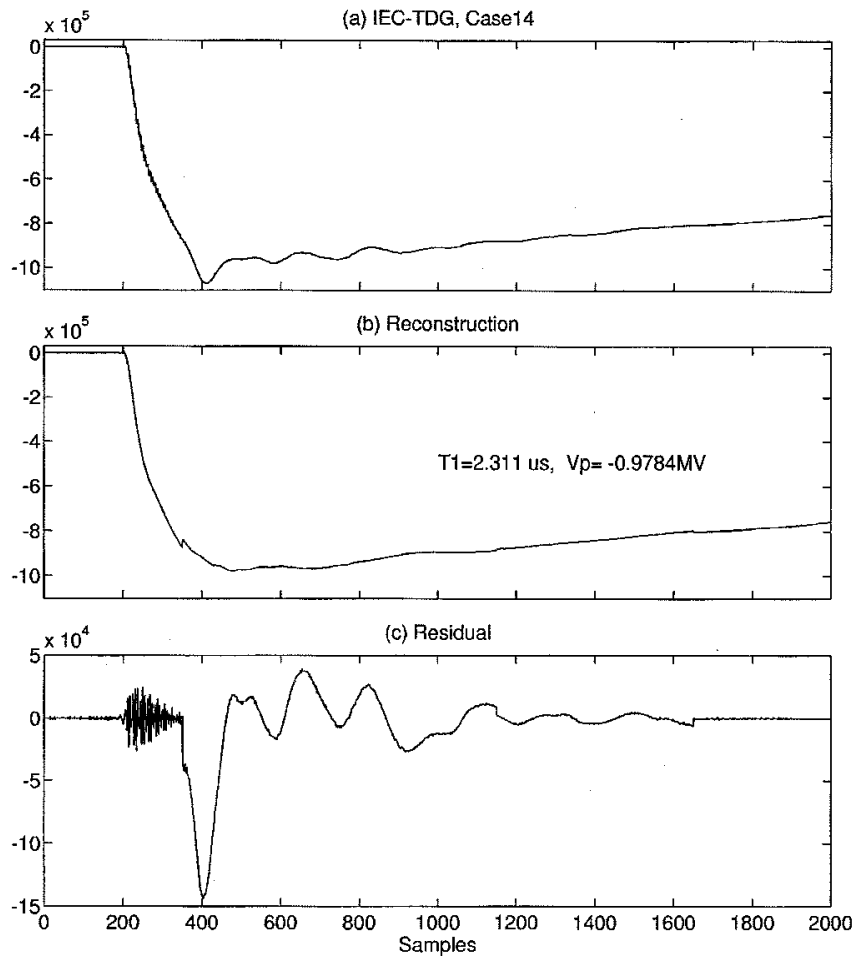

Fig. 6. Isolation of short-duration overshoot.

Another practically measured impulse (IEC-TDG, Case 14) with short-duration oscillation and noise on wavefront is shown in Fig. 6(a). The mean-curve is depicted in Fig. 6(b) and the residual is shown in Fig. 6(c). As can be seen, the mean-curve obtained is good. The simultaneous removal of high and low frequency components is depicted in these examples and the 
(a) Input
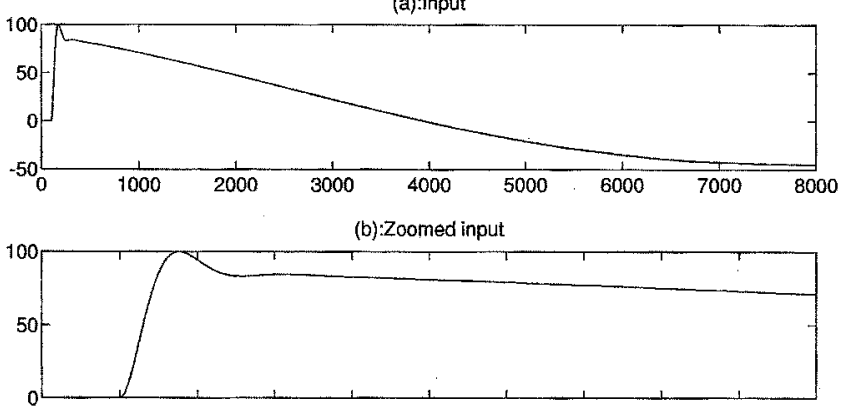

(c):Reconstruction

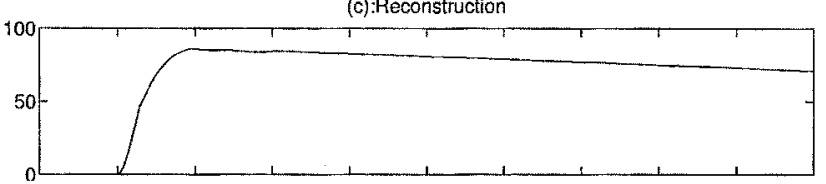

(d):Residue

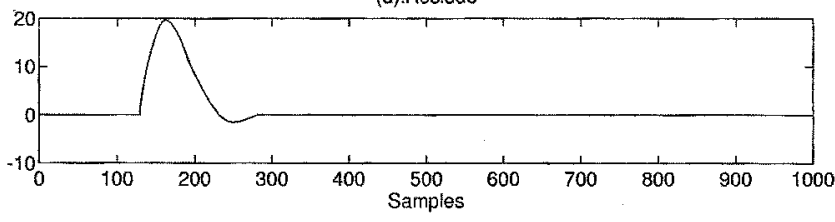

Fig. 7. Isolation of overshoot.

obtained impulse parameters are also shown. They deviate from the stipulated values slightly.

In this regard, it must be mentioned that both Cases 13 and 14 are practical impulses and the so-called virtual front-time and its limits specified in the IEC standard has been obtained as the mean of the results of the participating laboratories (mostly based on model-based curve fitting). Hence, the deviations in impulse parameters obtained by MSD must not be considered significant.

Next, consider an analytically computed waveform that is typical when impulse testing a low inductance winding of a transformer, shown in Fig. 7(a). The equation of the waveform is given below, where the voltage $v$ is in $\mathrm{kV}$ and time $t$ in microseconds.

$$
\begin{aligned}
v= & 101.9 \exp ^{-.00355 t} \sin (.0138 t+1.815) \\
& +115 \exp ^{-.977 t} \sin (1.636 t+4.177) .
\end{aligned}
$$

It has a characteristic overshoot on the front and an oscillating under-swing. The purpose of choosing this waveform is to illustrate that such waveforms can also be handled. The results obtained are depicted in Fig. 7(b)-(d). A study of the residue [Fig. 7(d)] has to be made to determine how the waveform parameters are to be calculated as per standards.

Different examples of full and chopped lightning impulse voltages containing noise, oscillations and overshoot were considered for mean-curve determination using the MSD approach. It must be emphasized that MSD method naturally allows easy identification of oscillations and over-shoot, and their processing as per the rules prescribed in IEC and that no changes in rules are called for at present. Both measured and mathematically generated data have been considered. It was observed that MSD method has, in principle, the capability of determining mean-curve from all types of impulses. It is important to summarize at this stage, some of the advantages of this approach:

1) Local smoothing or simultaneous removal of high and low frequency disturbance components and artifacts in the waveform is possible from selected time zones.

2) Removal of disturbances does not affect the rest of the waveform.

3) In contrast, conventional digital filtering does introduce end-effects and, more importantly, filters the entire waveform and processing of selected portions of the signal is not straightforward.

4) It can be seen that MSD approach can take into account all the present stipulations regarding frequency and amplitude of superposed oscillations or duration and magnitude of overshoot easily. Hence, there is no need for rewriting the standards regarding these matters to specifically deal with digital data.

Angrisani and others [9] have also reported a wavelet approach. In this, the input is first decomposed by an entropy approach, followed with a correlation criterion based on a model-based reference curve to reconstruct the meancurve. Finally, it is stated, further research is required to enhance results for some waveforms listed in IEC 61083 -2. In comparison, results presented in this paper have systematically treated all categories of waveform variations usually encountered in a uniform way. In perspective, some of the significant differences are:

* The use of entropy-based criterion does in no way simplify the task of identifying the components to be selected for reconstruction. Although, entropy criterion is an accepted signal processing approach, it has little significance in the. physics of impulse breakdown phenomenon.

* Further, use of reference curves (model-based) to select components for reconstruction defeats the basic philosophy of the approach.

* Since, a decision about the input being a standard impulse or not solely depends on the residual curve, inability to obtain the correct residual-curve from [9] is a serious drawback. In contrast, the approach in this paper separates individual artifacts superposed on the impulse, and thus aids in easy interpretation of IEC $60060-1$.

* Results in [9] show large-scale distortions at the two ends of signal. This will pose difficulties in automatic evaluation of impulse parameters. These distortions are not seen in the MSD approach.

Anticipating the above mentioned drawbacks, this paper has overcome them, while making it interactive and ensuring that it yields the residual and mean-curves. Lastly, it does not call for any requirement for revision of the existing standards.

\section{CONCLUSION}

A new method for estimation of mean-curve of impulse waveforms superposed by noise, oscillations and overshoot based on multiresolution signal decomposition has been proposed. This 
method does not assume any model for fitting the data to determine the mean-curve. Also, it provides simultaneous time and frequency information of the signal which is very useful to decide whether or not to remove an oscillation. Noise removal is inherent and needs no additional processing. The operation is interactive and found to be applicable to both full and chopped impulses. The method has been tested with typical data sets (considering most of the artifacts practically encountered) used in international intercomparisons. Results obtained indicate the potential of this approach; however, further use with practical data can reveal its deficiencies, if any.

\section{ACKNOWLEDGMENT}

L. Satish would like to thank Prof. M. Aro, Director of H. V. Institute, H. U. T., Finland, for providing excellent facilities and support.

\section{REFERENCES}

[1] High Voltage Test Techniques-Part I: General Definitions and Test Requirements, IEC 60-1 Standard, 1989.

[2] Standard Techniques for High Voltage Testing, IEEE Standard 4.

[3] T. R. McComb and J. E. Lagnese, "Calculating the parameters of full lightning impulses using model-based curve fitting," IEEE Trans. on Power Delivery, vol. 6, no. 4, pp. 1386-1394, Oct. 1991.

[4] Y. M. Li, J. Kuffel, and W. Janischewskyj, "Exponential fitting algorithms for digitally recorded high voltage impulse parameter evaluation," IEEE Trans. on Power Delivery, vol. 8, no. 4, pp. 1727-1735, Oct. 1993.

[5] J. Perez and J. Martinez, "Kalman filter algorithm for digitally recorded lightning impulse parameter evaluation," IEEE Trans. on Power Delivery, vol. 11, no. 4, pp. 1713-1719, Oct. 1996.

[6] _ , "Digitally recorded lightning impulse with overshoot parameter evaluation by using the Kalman filtering method," IEEE Trans. on Power Delivery, vol. 13, no. 4, pp. 1005-1014, Oct. 1998.

[7] C. P. Dias, A. M. Moura, and A. A. Vale, "Digital filtering of HV impulse tests results," in 7th International Symposium on High Voltage Engineering, Dresden, Aug. 26-30, 1991, paper \#62.08, pp. 121-124.
[8] V. R. Oliveria and G. C. Miranda, "Application of digital filter to lightning impulse parameters evaluation," in 10th International Symposium on High Voltage Engineering, Montreal, Aug. 25-29, 1997, pp. 431-434.

[9] L. Angrisani et al. " "A wavelet based approach for measurements on high voltage impulses," in 11th International Symposium on High Voltage Engineering, vol. 1, London, Aug. 22-27, 1999, pp. 205-208.

[10] F. Garnacho et al., "Evaluation procedure for lightning impulse parameters in case of waveforms with oscillations and/or an overshoot," IEEE Trans. on Power Delivery, vol. 12, no. 2, pp. 640-649, Apr. 1997.

[11] K. Schon et al., "International comparison of software for evaluating high voltage impulses and step responses," in 8th International Symposium on High Voltage Engineering, Yokohama, Japan, Aug. 23-27, 1993, paper \#51.01, pp. 289-292.

[12] L. Satish, "Short-time Fourier and wavelet transforms for fault detection in power transformers during impulse tests," IEE Proceedings-Science Measurement Technology, vol. 145, no. 2, pp. 77-84, Mar 1998.

[13] S. K. Pandey and L. Satish, "Multiresolution signal decomposition: A new tool for fault detection in power transformers during impulse tests," IEEE Trans. on Power Delivery, vol. 13, no. 4, pp. 1194-1200, Oct. 1998.

L. Satish was born in 1964 and received his Ph.D. from Indian Institute of Science (IISc), Bangalore, in 1993. He was in ETH, Zurich, from 93-95 pursuing post-doctoral research. Since May 1995, he is an Assistant Professor in the Department of High Voltage Engineering. His research areas are application of signal processing to H.V. testing and diagnostics, partial discharges measurements and pattern recognition. During 1998 summer, he was a Visiting Researcher at the High Voltage Institute, Helsinki University of Technology, Finland. He is a Member of TF 1.1 of the CIGRE WG 33-03.

B. I. Gururaj was born in 1933 and received his Ph.D. (1964) from Indian Institute of Science (IISc), Bangalore. After a short period of work in an Electrical Utility, he joined the Department of High Voltage Engineering, IISc, in 1960, and retired as Professor, in 1993. He has actively participated in the activities of CIGRE SC 33 and its working groups, as well as IEC TCs 28 and 42 and its working groups. He was Chairman of BISs committees on $\mathrm{H}$. V. techniques and surge arresters. He is presently an Independent Consultant. 